\title{
Experimental and Theoretical Study of Stripe Magnetic Domain Structure Drift in Iron Garnet Crystals
}

\author{
L.A. Pamyatnykh*, G.A. Shmatov, S.E. Pamyatnykh, M.S. Lysov, D.S. Mekhonoshin \\ AND A.V. DRUZHININ
}

Ural Federal University, Lenin Av. 51, Yekaterinburg, 620083, Russia

\begin{abstract}
The results of experimental and theoretical study of magnetic domain structure drift in low frequency oscillating magnetic field oriented perpendicular to the sample plate are presented. Experimental study was performed on uniaxial iron garnet $(\mathrm{TbErGd})_{3}(\mathrm{FeAl})_{5} \mathrm{O}_{12}(111)$ plate with rhombic anisotropy for the case when orientation of domain walls of stripe domains is preserved. Dynamic domain structure was revealed by means of magnetooptic Faraday effect and registered by high speed digital camera at the speed equal to $1200 \mathrm{fps}$. Theoretical model based on the motion equations for coupled harmonic oscillators that takes into account attenuation and field inhomogeneity along the plate is proposed.
\end{abstract}

DOI: 10.12693 /APhysPolA.127.388

PACS: $75.40 . \mathrm{Mg}, 75.78 . \mathrm{Fg}, 75.60 . \mathrm{Ch}$

\section{Introduction}

The drift of domain walls (DW) is a translational motion of an array of stripe domains as a whole in direction, perpendicular to the domain walls plane, in oscillating magnetic field. In Ref. [1] a dislocation mechanism of drift was proposed. In Ref. [2] it was suggested that translational motion of domain structure (DS) is caused by various inhomogeneities that lead to different conditions on the neighboring DW. Analytical theory of drift was proposed in [3].

In Ref. [4] experimental dependencies of drift speed in iron garnet single crystals with complex character of magnetic anisotropy on amplitude and frequency of external oscillating magnetic field were established. In Ref. [5] the drift of stripe domain structure was obtained by numerical simulation of vibrational motion of DW system modelled by the system of motion equations with non-homogeneous initial conditions for neighboring DW for frequencies that are close to natural vibration frequency of the DW system.

In Refs. [6, 7] numerical simulation of DW motion was performed for frequencies around $10^{2} \mathrm{~Hz}$, where the drift is observed experimentally, and dependencies of DW oscillations amplitude on amplitude of external magnetic field for the process of DW drift with reorientation of DW were presented.

In this work the results of experimental study of the drift in uniaxial (111) plate with rhombic anisotropy cut from iron garnet crystal are presented. The character of oscillations of DW of stripe domains is studied in the region of amplitudes of oscillating external magnetic field that includes the drift of the DS as a whole for the case when stripe domain DW orientation is preserved.

*corresponding author; e-mail: Lidia.Pamyatnykh@urfu.ru
A model of stripe domain structure motion that includes attenuation associated with attenuation of magnetization precession in magnetic field is proposed. In contrast to the earlier results of [5-7], it is shown that the drift of stripe domain structure takes place in the model in case of homogeneous initial conditions and proceeds despite the presence of attenuation.

\section{Experimental results}

Experimental study was performed on (111) iron garnet $(\mathrm{TbErGd})_{3}(\mathrm{FeAl})_{5} \mathrm{O}_{12}$ plate in an alternating magnetic field $H=H_{0} \sin (2 \pi f t)$ applied perpendicular to the plane of the sample. Basic parameters of the sample: thickness $L=48 \mu \mathrm{m}$, saturation magnetization $M_{\mathrm{s}}=40 \mathrm{Gs}$, uniaxial anisotropy constant $K_{\mathrm{u}}=3.8 \times 10^{3} \mathrm{erg} / \mathrm{cm}^{3}$, cubic anisotropy constant $K_{1}=-3.2 \times 10^{3} \mathrm{erg} / \mathrm{cm}^{3}$. Exchange interaction constant $A \approx 10^{-7} \mathrm{erg} / \mathrm{cm}$, attenuation parameter $\alpha=0.45[8]$. DS was detected using magnetooptic Faraday effect and recorded by high speed digital camera at the speed equal to $1200 \mathrm{fps}$.

Upon application of alternating magnetic field with frequency $f=150 \mathrm{~Hz}$ the oscillations of domain walls were observed. With increase of the field amplitude $H_{0}$ to 100 Oe the oscillations were superimposed by translational motion of the stripe domain structure in a direction, perpendicular to the domain walls.

The dependencies of DW coordinates and coordinates of the center of the domain on time were determined (Fig. 1). The center of the domain (Fig. 1, curve 3) moves translationally with average speed equal to $0.16 \mathrm{~mm} / \mathrm{s}$. The neighboring domain walls (Fig. 1, curves 1, 2) oscillate in antiphase with the frequency of external magnetic field and simultaneously move translationally with the speed of the center of the domain. Solid lines in Fig. 1 are approximations of the corresponding points by the sum of harmonic and linear functions.

Figure 2 shows the dependence of DW oscillations amplitude (Fig. 2, curve 1) and dependence of the speed 


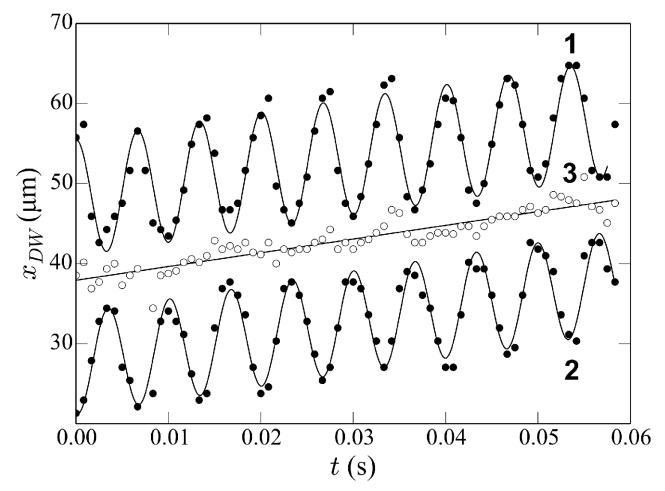

Fig. 1. Coordinates of the domain walls (curves 1, 2) and of the center of the domain (curve 3) for 9 oscillation periods of the external magnetic field. Experimental results: $f=150 \mathrm{~Hz}, H_{0}=111$ Oe. Solid curves are approximations to experimental points.

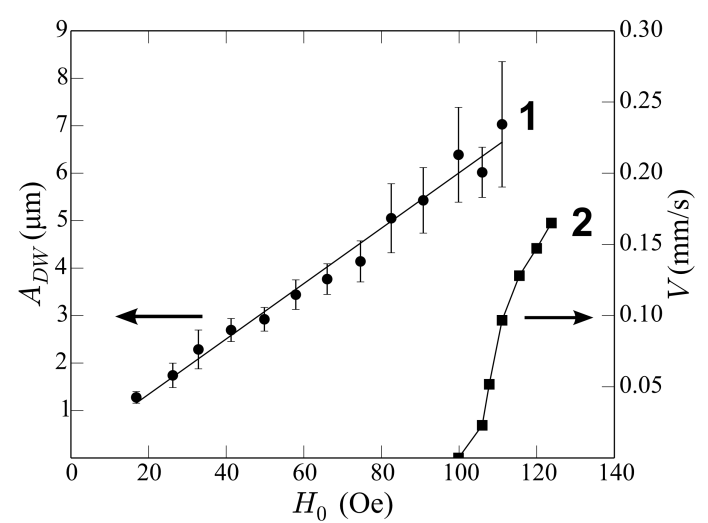

Fig. 2. Dependencies of amplitude $A_{\mathrm{DW}}$ of DW oscillations (curve 1) and of DW drift speed $V$ (curve 2) on amplitude of external magnetic field $H_{0}$. Experimental results: $f=150 \mathrm{~Hz}$.

of drift (Fig. 2, curve 2) on amplitude of external oscillating magnetic field. The amplitude of oscillations of DW appears to be a linear function of $H_{0}$ in the interval of amplitudes that includes the drift of the domain walls. The observed threshold field of start of the drift $H_{0}=100 \mathrm{Oe}$ is due to rapid increase of the number of magnetic dislocations [1] that nucleate in the sample at fields $H_{0}>100$ Oe.

In the investigated sample the orientation of DW maintained throughout the interval of existence of the drift of stripe domains, which is due to the relatively high value of rhombic anisotropy constant $K_{p}\left(K_{p} / K_{u}=1.2\right)$. While at $K_{p} / K_{u}=0.6$ the drift was accompanied by reorientation of the domain walls [7].

\section{Numerical simulations}

DW motion is described by the equations of forced oscillations of a system of coupled harmonic oscillators (see, e.g., [9]). It is assumed that internal structure of DW does not change during the motion, DW has effective mass and moves due to pressure forces acting on its surface.
Consider a model of stripe domain structure in a planeparallel uniaxial plate with thickness $L$ in external magnetic field $H(t)=H_{0} \sin (\omega t)$, that is applied perpendicular to the plane of the sample and parallel to the $O Z$ axis (Fig. 3).

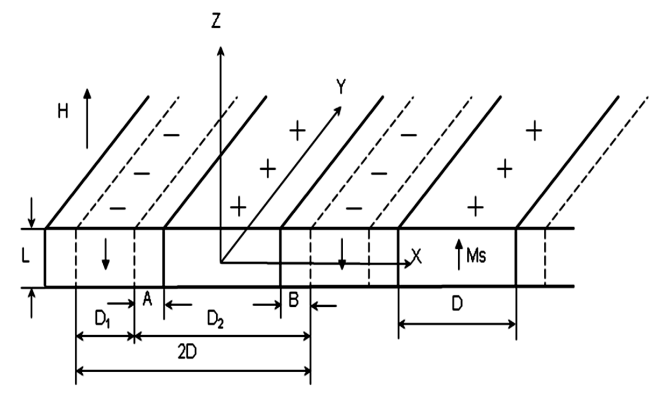

Fig. 3. The model of stripe domain structure.

In this case the following forces are acting on the DW: external oscillating magnetic field force that shifts the DW from equilibrium, "restoring" force associated with magnetostatic energy of stripe domain structure and dynamic friction force associated with attenuation of magnetization precession in magnetic field. Coercivity of domain walls is not considered in this model.

The easy axis of magnetization is perpendicular to the sample plate and parallel to the $O Z$ axis. Magnetization vectors $\boldsymbol{M}_{\mathrm{S}}$ in the domains are oriented either along the $O Z$ axis or in the opposite direction. The period of the domain structure is equal to $2 D$, where $D$ is the width of a single domain.

Homogeneous oscillating magnetic field will only cause oscillations of DW in the model without translational motion of the domains. In non-homogeneous case when, for example, the amplitude of oscillating field is nonhomogeneous, the forces that act on the walls of a single domain will be different, which can cause the drift of DS. Similar effect occurs in case when additional inhomogeneous constant magnetic field is applied to the sample or in case of inhomogeneous magnetization in the plate's plane. In the simplest case of weak gradient inhomogeneity, i.e. when the inhomogeneity is described by a linear function of coordinate, the difference of forces that act on the domain walls is proportional to the distance between the DW. For stripe domain structure this will correspond to the force that acts on the domain and does not depend on the coordinate.

Denote the displacements of domain walls from their position in the absence of magnetic field by $A$ and $B$. Such displacements are equal for all periods of the domain structure at any given moment of time. Thus, for every period $D_{1}=D-A-B, D_{2}=D+A+B$.

The symmetry of the problem allows to write down the motion equations for two neighboring domain walls, which is sufficient to describe the motion of all domain walls in the given model of domain structure. The energy densities of external magnetic field $\gamma_{H}$ and of magnetostatic energy $\gamma_{m}$ have the form given in [7] (derivation is given in [10]). 
In this case the equations that describe the DW motion of stripe DS in direction perpendicular to the DW can be written in the following form (in dimensionless variables):

$$
\left\{\begin{array}{l}
\frac{\partial^{2} a}{\partial \tau^{2}}+\eta \frac{\partial a}{\partial \tau}+f(a, b)+\Delta-\pi h \sin (\nu \tau)=0, \\
\frac{\partial^{2} b}{\partial \tau^{2}}+\eta \frac{\partial b}{\partial \tau}+f(a, b)-\pi h \sin (\nu \tau)=0,
\end{array}\right.
$$

where

$$
\begin{aligned}
& f(a, b)=\frac{a+b}{2}-\frac{2}{\ell} \sum_{n=1}^{\infty} \\
& \quad \times\left[\frac{(-1)^{n}}{n^{2}}[1-\exp (-n \ell)] \sin \frac{n(a+b)}{2}\right],
\end{aligned}
$$

$a=2 \pi A / D$ and $b=2 \pi B / D$ are dimensionless variables, that correspond to the displacements of left and right domain walls, respectively, $\ell=\pi L / D, h=H_{0} / 4 \pi M_{\mathrm{s}}$, $\omega=2 \pi f, \nu=\omega / \Omega, \tau=t \Omega, \Omega^{2}=8 \pi M_{\mathrm{s}}^{2} / m D$, $m$ is an effective mass of DW per unit area, $\eta=$ $\alpha\left(D /\left(A / K_{\mathrm{u}}\right)^{1 / 2}\right)^{1 / 2} \approx 8.9$ is a dimensionless attenuation parameter.

Constant term $\Delta$ in the first Eq. (1) corresponds to the difference of forces that act on the domain walls.

The system was solved using Wolfram Mathematica (c) (LSODA solver) [11] with homogeneous initial conditions. Figure 4 shows the results of numerical solution of the system (1) with parameters of the real sample (see Sect. 2). For these values of parameters $m \approx 1 \times 10^{-10} \mathrm{~g} / \mathrm{cm}^{2}, \Omega \approx 4.5 \times 10^{8} \mathrm{~Hz}$.

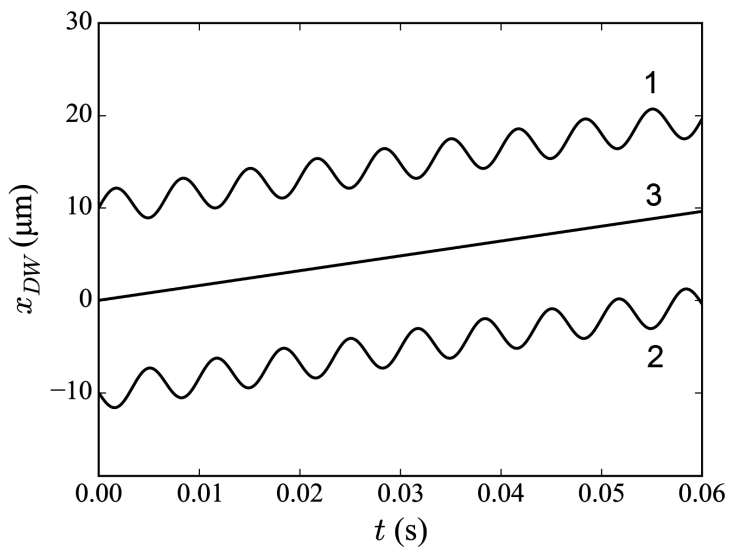

Fig. 4. Coordinates of the DW (curves 1,2) and of the center of the domain (curve 3 ) for 9 oscillation periods of the external magnetic field. Numerical simulation: $f=150 \mathrm{~Hz}, H_{0}=111 \mathrm{Oe}$.

Curves 1 and 2 in Fig. 4 show the positions of the right and left DW with respect to time, curve 3 shows the position of the center of the domain. The figure shows that DW movement is oscillatory and the period of DW oscillations is determined by the period of oscillations of external magnetic field. The average speed of the DS drift is determined by the inhomogeneity parameter $\Delta$. The value of $\Delta=2 \times 10^{-6}$ corresponds to the experimentally observed speed of drift equal to $0.16 \mathrm{~mm} / \mathrm{s}$. The change of sign of $\Delta$ results in the change of the direction of the drift to the opposite.

\section{Conclusions}

A threshold nature of stripe DS drift is established experimentally. It is shown experimentally that DW maintain their orientation in the whole interval of drift of stripe domains at high level of rhombic anisotropy $\left(K_{p} / K_{u}=1.2\right)$ and DW oscillations amplitude depends linearly on the amplitude of external magnetic field including the interval of DW drift.

The results of numerical simulations, that take into account attenuation and inhomogeneity of a simple type along the plate, are in agreement with experimentally observed DS behavior: neighboring DW oscillate in antiphase while the DS as a whole drifts with constant speed. Comparison of computed drift speed with experimentally observed value allowed to estimate the parameter of inhomogeneity of the field.

The drift in the experiment can be caused by other physical reasons as well. Full consideration of the matter is a subject of further work.

\section{Acknowledgments}

The results of this research were obtained within the state assignment of the Russian Ministry of Education and Science. This research project has been supported by UrFU under the Framework Programme of development of UrFU through the "Young scientists UrFU" competition.

\section{References}

[1] L.A. Pamyatnykh, M.S. Lysov, G.S. Kandaurova, Bull. Russ. Acad. Sci. Phys. 71, 1497 (2007).

[2] Yu.N. Dragoshanskii, Ye.V. Khan, V.A. Zaikova, Fiz. Met. Metalloved. 39, 289 (1975).

[3] V.G. Bar'yakhtar, Yu.I. Gorobets, S.I. Denisov, JETP 98, 1345 (1990).

[4] L.A. Pamyatnykh, M.S. Lysov, G.A. Shmatov, G.S. Kandaurova, A.V. Druzhinin, Bull. Russ. Acad. Sci. Phys. 74, 1417 (2010).

[5] M.M. Solov'ev, B.N. Filippov, Phys. Met. Metallogr. 98, 241 (2004).

[6] L.A. Pamyatnykh, A.V. Druzhinin, M.S. Lysov, S.E. Pamyatnykh, G.A. Shmatov, Bull. Russ. Acad. Sci. Phys. 77, 1231 (2013).

[7] L.A. Pamyatnykh, G.A. Shmatov, M.S. Lysov, S.E. Pamyatnykh, D.S. Mehonoshin, in: Solid State Phenomena 215, 437 (2014).

[8] Y.M. Yakovlev, S.S. Gendelev, Ferrite Monocrystals in Electronics, Soviet Radio, Moscow 1975.

[9] S. Chikazumi, Physics of Ferromagnetism, Clarendon Press, Oxford 1997.

[10] G.S. Kandaurova, L.G. Onoprienko, The Main Problems of the Theory of Magnetic Domain Structure, UrGU, Sverdlovsk 1977.

[11] A.C. Hindmarsh, ODEPACK, A Systematized Collection of ODE Solvers, IMACS Transactions on Scientific Computation, Vol. 1, Scientific Computing, North-Holland, Amsterdam 1983, p. 55. 